\title{
Biomarkers for the detection of graft-versus-host disease in cancer patients after bone marrow transplantation
}

This article was published in the following Dove Press journal:

Current Biomarker Findings

7 August 2012

Number of times this article has been viewed

\author{
Sophie Paczesny \\ Department of Pediatrics, University \\ of Michigan, Ann Arbor, MI, USA
}

\begin{abstract}
Allogeneic hematopoietic stem cell transplantation (HSCT) is the most effective form of tumor immunotherapy available to date, and the frequency of transplants continues to increase worldwide. However, while allogeneic HSCT can induce beneficial graft-versustumor (GVT) effects, the adverse effect of graft-versus-host disease (GVHD), which is closely linked to GVT, is the major source of morbidity and mortality following HSCT. Acute GVHD (aGVHD) develops when the donor's immune cells from the graft attack the patient's skin, liver, or intestines. Target organs involved in chronic GVHD (cGVHD) are variable, resemble autoimmune manifestations, and typically include skin; mucosa (mouth, eyes, genitalia); muscles/ fascia/joints; lungs; and gastrointestinal tract/liver. GVHD occurs in two forms, classically distinguished as aGVHD, beginning before day 100 after HSCT, and cGVHD, occurring after day 100, although it is now accepted that these are two separate pathophysiologic entities. Currently available diagnostic and staging tools frequently fail to identify those at higher risk of GVHD progression, unresponsiveness to different forms of therapy, or death. Furthermore, there are shortcomings in the prediction of the future occurrence of GVHD before clinical signs develop. In parallel, in recent years there has been an explosive evolution of proteomics technologies, largely due to important advances in chemistry, engineering, high-throughput technical devices, and bioinformatics. Building on these opportunities, plasma biomarkers have been identified and validated both as promising diagnostic tools of GVHD and as prognostic tools for nonrelapse mortality. These biomarkers might facilitate timely and selective therapeutic intervention. However, such biomarkers should be more widely validated and incorporated into a new grading system to risk-stratify patients and better customize treatment. This review identifies biomarkers for the detection of GVHD; summarizes the current information on available GVHD biomarkers, which are mostly aGVHD biomarkers; proposes future prospects for the blinded evaluation of these biomarkers in samples collected as part of a multicenter prospective study; and discusses the need for biomarkers of cGVHD.
\end{abstract}

Keywords: GVHD, hematopoietic stem cell transplantation, risk stratification, proteomics

\section{Introduction}

Allogeneic hematopoietic stem cell transplantation (allo-HSCT) is an increasingly widely used therapy in a range of malignant and nonmalignant hematologic diseases. In allo-HSCT, the host immune and bone marrow systems are replaced by the donor immune and hematopoietic stem cells, with both positive and negative consequences. In malignant disease, the donor immune system can recognize residual tumor cells as foreign and eradicate them by immunological means, called the graft-versus-tumor (GVT) effect. Unfortunately, donor immune cells may also attack normal host tissue,
Correspondence: Sophie Paczesny University of Michigan Comprehensive Cancer Center 6303,I500 E Medical

Center Dr, Ann Arbor,

MI 48I09-5942, USA

$\mathrm{Tel}+\mathrm{I} 7346155283$

Fax + I 734647927 |

Email sophiep@med.umich.edu
(C) 2012 Paczesny, publisher and licensee Dove Medical Press Ltd. This is an Open Access article which permits unrestricted noncommercial use, provided the original work is properly cited. 
particularly the skin, liver and gastrointestinal (GI) tract, resulting in the graft-versus-host disease (GVHD) effect. The occurrence of GVHD remains one of the major barriers to more widespread and successful application of allo-HSCT. Classically, GVHD was distinguished into two forms: acute, which is defined as that arising before day 100 post-HSCT, and chronic, which occurs after that time. ${ }^{1,2}$ However, this definition was updated by the new National Institutes of Health $(\mathrm{NIH})$ classification that includes late-onset acute GVHD (aGVHD) (after day 100) and an overlap syndrome with features of both the acute and chronic disorders. ${ }^{3,4}$ These new forms of GVHD can be explained by the wider utilization of HSCT in older recipients being transplanted using reduced-intensity conditioning regimens.

A major barrier to GVHD research and treatment is that the diagnosis and prognosis rely almost entirely on the presence of clinical symptoms, which are sometimes confirmed by biopsy. Currently, no laboratory tests exist to predict the risk of developing GVHD, responsiveness to treatment, or patient survival. This absence of validated biomarkers for GVHD is partly due to the complex pathology of GVHD, which involves both soluble and cellular factors. GVHD can be considered in a framework of three distinct sequential phases of immune system cellular activation and cytokine production, which can influence specific cellular and protein levels in the GVHD patient's blood. ${ }^{5}$ This three-step model involves: (1) conditioning regimen-related damage and the release of endotoxins such as lipopolysaccharide, (2) donor $\mathrm{T}$ cell proliferation and differentiation in response to residual host antigen-presenting cells, and (3) target organ damage by effector cells. The importance of biomarkers in HSCT settings is crucial, as the ability to identify patients at high risk for GVHD early in their transplant and treatment course has important therapeutic consequences, including more stringent monitoring and/or preventative care. The ability to identify patients who will not respond to traditional treatment and who are at particularly high risk for subsequent morbidity and mortality could result in tailored treatment plans such as additional immunosuppressive treatments that might be more effective if introduced early for high-risk patients. Equally important is the identification of patients who will respond well to treatment, which may allow for more rapid tapering of steroid regimens, thereby reducing long-term toxicity in low-risk patients.

The current review will provide an update on the discovery and validation of the most clinically relevant biomarkers of aGVHD and insights for specific recommendations on their use in clinical trials.

\section{Biomarkers: some useful definitions}

The NIH Biomarkers Definition Working Group has defined a "biomarker" as "a characteristic that is objectively measured and evaluated as an indicator of normal biologic processes, pathogenic process, or pharmacologic responses to a therapeutic intervention." ${ }^{\circ}$

There are three types of biomarkers: (1) disease biomarkers that give clues to the pathophysiology of a disease (or type 0 ), (2) biomarkers to detect drug effects (type I), or (3) biomarkers as surrogate end points in clinical trials (type II).

Subcategories of disease biomarkers (type 0) are diagnostic biomarkers that will allow for early detection of a disease, follow-up biomarkers that track the disease progression over time, prognostic biomarkers for prognostic evaluation of outcomes of the disease, and predictive biomarkers to predict disease at the presymptomatic stage associated with a risk for a disease and as a candidate for a screening test. This is the type of biomarker detailed in this review. This subcategory also includes biomarkers for drug discovery, as either disease biomarkers as targets for drug discovery or biomarkers of the interaction of a drug with its therapeutic target.

Subcategories of biomarkers to detect drug effects (type I) include efficacy biomarkers indicating beneficial effects of a drug, mechanism biomarkers that report a downstream effect of a drug, and toxicity biomarkers that report toxicological effects of a drug. Examples of type I biomarkers would include glucose lowering for a diabetes drug, decreased target phosphorylation after a kinase inhibitor, or viral load as a function of survival benefit for anti-human immunodeficiency virus therapy.

Biomarkers as surrogate end points in clinical trials (type II) are substitute measures for clinical outcomes. Classical surrogate endpoints are arterial blood pressure reduction as a surrogate for reduced stroke and cardiovascular mortality, low-density lipoprotein-cholesterol reduction for reduced cardiovascular mortality, and prolonged QT interval for risk of sudden cardiac death.

\section{Ideal biomarkers for GVHD}

The need for biomarkers post-HSCT is due to the limitations of current predictors. Known risk factors pre-HSCT are related to genetic factors, including human leukocyte antigen (HLA) disparities between donor and recipient, age, unrelated transplant, conditioning regimen intensity, malignant disease status, and donor graft content. A diagnosis of aGVHD post-HSCT relies entirely on clinical signs in one of three major target organs: skin, liver, and/or GI tract ${ }^{7}$ and can be 
confirmed by biopsies of these organs. However, histologic severity on biopsy has not been consistently correlated with clinical outcome. ${ }^{7}$ Currently, there are no validated simple tests to diagnose, prognose, or predict GVHD. A good diagnostic GVHD test will be able to distinguish patients with GVHD from those without the disease (eg, GI GVHD vs infectious colitis, skin GVHD vs drug rash). Furthermore, from a practical point of view, the biomarker would (1) specifically and sensitively reflect the disease state; (2) be noninvasive, such as a blood or urine test; and (3) be rapid, simple, accurate, inexpensive and standardized. Ideally, the same test should also be prognostic for aGVHD outcomes such as the response to GVHD treatment, the survival, or nonrelapse mortality (NRM). This test will then allow for early risk stratification before initiation of treatment. A biomarker that will allow for prediction of aGVHD prior to clinical signs will have tremendous impact in this disease and will allow for preemptive interventions. The ideal biomarker for GVHD should differentiate between GVHD and the GVT effect.

Thus, the current invasive procedures used, such as biopsies of target organs, should not be the preferred diagnostic and prognostic indicators. Indeed, endoscopic biopsy is often used to confirm the diagnosis but, besides the fact that it is an invasive procedure, histologic severity on biopsy has not consistently correlated with clinical outcome. In the clinic, short duration and robust assays are preferred. Several new approaches, such as proteomics, may lead to the discovery of new candidate molecules, but the ultimate blood test should be based on immunoassays.

\section{Single versus multiple biomarkers of GVHD}

While several biomarkers exist, in most cases none is sufficiently sensitive or specific on its own for either a diagnostic or predictive test. Thus, the simultaneous use of several markers may increase specificity, predictive, or diagnostic performance. ${ }^{8}$ To create a comprehensive GVHD biomarker panel, proportional odds logistic regression models are used to determine a composite panel that will generate a receiver operating characteristic curve with the area under the curve being less than 0.8 , meaning that $80 \%$ of the positive results are true positives. Presumably, for an aGVHD diagnosis, a combination of tissue-specific and systemic biomarkers will be more informative than individual markers. However, if a biomarker is not highly correlated to other biomarkers or clinical predictors, one or two biomarkers could be sufficient for either diagnostic or predictive tests. To evaluate the number of biomarkers that will give the most information, optimized classification models that simultaneously minimize the misclassification error rate and maximize the area under the curve are used. ${ }^{9}$

\section{Types of GVHD biomarkers Disparities between histocompatibility antigens}

Antigen disparity can be at the level of the major histocompatibility complex (MHC), or at the level of minor histocompatibility antigens (mHAs). The severity of aGVHD is directly related to the degree of $\mathrm{MHC}$ mismatch. ${ }^{10} \mathrm{In}$ bone marrow transplantations (BMTs) that are $\mathrm{MHC}$ matched but mHA disparate, donor $\mathrm{T}$ cells still recognize MHC peptide derived from the products of recipient polymorphic genes, the mHAs. ${ }^{11-13}$ The expression of mHAs is wide and variable. Thus, different mHAs might dictate variable phenotype, target organ involvement, and antitumor responses after allo-HSCTs. ${ }^{14}$ Some mHAs, such as HA-1, HA-2, HB-1 and BCL2A1, are primarily found on hematopoietic cells, whereas others, such as the H-Y antigens, HA-3, HA-8, and UDP-glucuronosyltransferase 2B17, are ubiquitous. ${ }^{15,16}$ These disparities are well-known risk factors before transplant and the goal of clinicians is always to best match the MHC disparities, although full match is not always possible and match for $\mathrm{mHA}$ disparities is not yet able to be performed. Furthermore, MHC disparities cannot be used to monitor GVHD after transplant and thus do not represent ideal biomarkers of GVHD.

\section{Non-HLA polymorphisms}

There is increasing evidence that non-HLA polymorphisms can influence the risk of aGVHD and chronic GVHD (cGVHD). Most of the genetic variation in humans consists of single nucleotide polymorphisms (SNPs) that produce functional differences in gene products. Several SNPs have been identified as risk factors for GVHD, such as tumor necrosis factor alpha (TNF $\alpha$ ), interleukin (IL)-6, interferon-gamma, IL-10, UDP-glucuronosyltransferase 2B17, and reviewed elsewhere. ${ }^{17}$ However, clinicians are confronted with the same issue as that for mHA disparities and selection of donors according to SNP genotyping is still not performed in clinic.

\section{MicroRNAs}

MicroRNAs (miRNAs) - small, mostly noncoding RNA gene products - are molecules derived from larger segments of precursor RNA. They are 21-25 nucleotide transcripts that repress gene function through interaction with target mRNAs. ${ }^{18}$ miRNAs target the control of gene activity at 
multiple levels, specifically transcription, translation, and protein degradation. Gene regulation mediated by miRNAs is guided by the base-pairing rules of Watson and Crick. ${ }^{19}$ Each miRNA is thought to regulate multiple genes, and since approximately 1000 miRNA genes have been identified in humans, the potential regulatory capacity of miRNAs is enormous and could be as important as that of transcription factors. Circulating miRNAs have recently been used as promising novel biomarkers in diverse diseases (reviewed in $\mathrm{Ajit}^{20}$ ), including GVHD. ${ }^{21}$

\section{Cellular biomarkers}

There are several different immune cell populations whose function and numbers are altered in GVHD. The potential to manipulate specific immune cell populations ex vivo and in vivo to modulate immune responses in GVHD patients will allow for development of new therapies. In addition, some of these immune cell subsets, particularly regulatory $\mathrm{T}$ cells, appear promising as biomarkers in $\mathrm{aGVHD}^{22-24}$ and cGVHD. ${ }^{25}$ Dendritic cells, monocytes, and gamma-delta $\mathrm{T}$ cells are also promising cellular subsets that could be used as markers post-HSCT. ${ }^{26,27}$ In cGVHD, B cells and their modulators such as B cell-activating factor are important biomarkers. $^{28}$

\section{Proteomic biomarkers}

"Proteomics," from "PROTEins expressed by a genOME," is the systematic analysis of the protein profiles of a sample. Unlike the genome, the proteome varies with time and is defined as the proteins present in one sample at a certain point of time. Thus, proteins represent ideal biomarkers in the posttransplant setting and they have been widely studied, which will be detailed further on in this review.

\section{Current technologies for detection of GVHD biomarkers}

\section{Bio-fluids of interest}

Ideal clinical tests are based on noninvasive collection, which allows for repetitive collection of samples from the same patient over a short period. GVHD biomarkers may be produced by several sources such as donor cells, the local or systemic cytokine milieu, or recipient target tissues during disease development. These proteins may then be released into a variety of body fluids. For noninvasive tests used for diagnostics or screening, bio-fluids such as plasma, sera, or urine are preferred as samples. Enormous effort has been made to develop standardized methods of clinical sample collection for proteomic studies. ${ }^{29,30}$ Plasma and sera are the most frequently analyzed bio-fluids. The levels of individual blood proteins represent a summation of multiple, disparate events that occur in every organ system. Plasma and sera contain proteins shed by the affected tissue as well as proteins that reflect secondary systemic changes. However, plasma and sera are highly complex mixtures containing high levels of many different proteins with a wide dynamic range, spanning 12 orders of magnitude from albumin to the lowest abundance. Often the most clinically relevant proteins, such as cytokines and their receptors, are the least abundant, ${ }^{31,32}$ making the study of the proteome complex. To detect these proteins of low abundance, both depletion of the predominant proteins and subsequent fractionation of the proteome are required.

Urine samples represent an alternative to plasma/sera samples for biomarker discovery. Urine has three main advantages over plasma/sera: (1) it can be obtained in large quantities, (2) the protein mixture is far less complex and the variation in protein abundance is low, ${ }^{33}$ and (3) it is more stable than plasma. ${ }^{34}$ However, since the proteins in urine are produced mainly from kidney function $(\sim 70 \%)$ and partially by glomerular filtration of plasma proteins $(\sim 30 \%)$, urine yields better information about diseases in the organs directly involved in its production and excretion, such as the kidneys, and is thus less informative for systemic diseases. ${ }^{33}$

\section{Proteomic technologies for biomarker discovery}

Advances in engineering have allowed for increased data throughput, enabling the study of complete sets of molecules ("-omics") with exponential speed, accuracy, and cost-effectiveness. Thus, analysis of the entire spectrum of molecular and cellular organization is now possible, enabling researchers to gain insight into the mechanism of disease, with fewer a priori assumptions. However, from genes $(\sim 20,000)$ to proteins, there are two more levels of complexity: the transcriptome ( 100,000 RNA transcripts) and the proteome $(\sim 1,000,000$ proteins). Here, focus is on the use of proteomics for the molecular diagnosis of GVHD post-HSCT, since proteins are more proximal than other cellular metabolites to the ongoing pathophysiology of a disease. ${ }^{35}$ Both nonmass spectrometry (MS)- and MS-based proteomic approaches have been employed to search for potential GVHD biomarkers.

\section{Antibody-based approaches}

Immunoassays are analytical tests that harness the unique properties of antibodies, thus extremely sensitive. 
They have proven to be one of the most productive technological contributions to medicine and fundamental life science research in the twentieth century. The unique characteristics of antibodies are derived from their three important properties: (1) their ability to bind to an extremely wide range of natural and manufactured chemicals, biomolecules, and cells, as antibody-binding sites are derived from a very large number of potential combinations of amino acid sequences; (2) exceptional binding specificity that enables the measurement of picomolar $\left(10^{-12}\right)$ amounts of proteins in blood samples; and (3) the strength of binding between an antibody and its target that makes the test accurate and precise, even at low concentrations. ${ }^{36}$ To screen for aGVHD biomarkers, antibody microarrays dotted with hundreds of antibodies have been employed, allowing hundreds of proteins in complex biological matrices to be measured. ${ }^{8}$ The advantages of immunoassays are that they are suited for the characterization of complex protein mixtures, such as human plasma; quantitative; highly sensitive for low-abundance proteins such as cytokines; and high throughput. However, only a restricted number of antibodies are on the array (thus, bias could be introduced) and there is high cross-reactivity between antibodies and nontarget proteins. ${ }^{37}$

\section{MS-based approaches}

The majority of nonantibody proteomic strategies are based on MS, which has become a powerful tool for characterizing and assessing both qualitative and quantitative changes in complex protein mixtures. ${ }^{38}$ Two types of MS techniques in clinical proteomics have been used, pattern profiling and detailed characterization of proteins. Pattern profiling compares polypeptide spectra obtained by matrix-assisted laser desorption/ionization time of flight (MALDI-TOF) MS, which is used to show which patients suffer from a particular disease without the identification of individual profile components. A variant of MALDI-TOF MS is surface-enhanced laser desorption/ionization time of flight (SELDI-TOF) MS, which combines MALDI-TOF MS with selective sample fractionation on modified surfaces placed directly on the sample target. ${ }^{39}$ These MS profiling methods do not require an in-depth analysis, thus are relatively high throughput. Consequently, they are less suitable for in-depth discovery approaches. Furthermore, because the factors influencing the final oligopeptide profiles of body fluid samples are so complex, MS profiling has not yet met the standards required in clinical practice. This technique has been applied in aGVHD research to screen biomarker candidates in both serum $^{40}$ and saliva. ${ }^{41}$
Other approaches rely on separation of protein samples followed by MS. The most frequently employed gel-based techniques for protein separation are two-dimensional (2D) polyacrylamide gel electrophoresis ${ }^{42}$ and $2 \mathrm{D}$ differential gel electrophoresis. ${ }^{43}$ Three-dimensional separation of proteins, differentially labeled with fluorescent dyes (Cy3 [green] and Cy5 [red]) according to their charge, hydrophobicity, and molecular mass, have been applied to aGVHD diagnoses ${ }^{44}$ and heart ischemic insult. ${ }^{45}$

Despite the utility of gel-based techniques, gel-free separation methods, such as liquid chromatography (LC) $)^{46,47}$ and capillary electrophoresis, ${ }^{48}$ have provided better separation because they overcome several limitations of gel separation, such as time consumption, poor separation of proteins with low or high molecular weight or an extreme isoelectric point, and difficult quantification of mixed spots. Gel-free techniques also offer the prospect of an easy workflow with a direct connection to the mass spectrometer. MS is the final step in the analytical procedure and enables both the reliable identification of proteins and determination of their isoforms and posttranslational modifications. MS allows unambiguous quantification, particularly when tandem MS (ie, MS/MS) is employed, ${ }^{49}$ and has been used most recently for quantification with either label-free methods or isotopically labeled tags. ${ }^{50-52}$ In addition, new instrumentation, such as the ultra-high resolution linear ion trap orbitrap mass spectrometer (eg, Orbitrap Elite, Thermo Scientific, Waltham, MA), facilitates top-down LC-MS/MS and versatile peptide fragmentation modes. ${ }^{53}$ The mass spectra are then matched to a sequence database to identify proteins. ${ }^{54}$

At present, these approaches are not suitable for validation purposes because of the time it takes to undertake them, but they remain the most efficient methods for biomarker discovery in clinical research.

\section{Proteomic approach for high- throughput validation of GVHD biomarkers}

Although proteomics holds great promise for biomarker development, gaps remain between biomarker discovery and biomarker validation. Indeed, the validation of biomarkers has obstacles of its own. Most noteworthy is the paucity of affinity-capture reagents, such as high-quality antibodies with the required affinities and specificities for the target, leading to a bias in the prioritization of candidate markers. Furthermore, the number of samples required for validation increases as the biomarker advances through each test phase, augmenting the need for high-throughput assays. 
The most applicable approach for the quantitation of individual proteins for validation remains the sandwich enzyme-linked immunosorbent assay (ELISA), which is highly specific and employs two antibodies specific for the candidate protein. The procedure is also relatively simple and highly reproducible from performer to performer and laboratory to laboratory, limiting both inter- and intra-assay variability. Finally, due to the urgent need for GVHD blood tests, Paczesny et $\mathrm{al}^{46,47}$ primarily tested proteins with available antibodies. The main disadvantage of validation by ELISA is the large volume of patient plasma required. Thus, multiplexing technologies are preferred if they show absence of cross-reactivity. ${ }^{37}$ Recently, multiple reaction monitoring has emerged as a potentially useful technique for clinical diagnostics. ${ }^{55}$ This rapid tandem mass spectrometric technique enables the targeted monitoring and quantification of candidate molecules in complex samples.

\section{Identified and validated GVHD biomarkers}

GVHD is not only a systemic immunological disorder; it also affects specific organ systems, including the skin, GI tract, and liver. The following reviews systemic and target-specific aGVHD biomarkers and their diagnostic and prognostic values, as well as how these were identified, whether by using the pathology of aGVHD or via a proteomic discovery approach. If the workflow did not include proteomic discovery, only studies with a sample size larger than 40 patients were reviewed. Table 1 summarizes these studies.

\section{Systemic biomarkers of aGVHD}

Due to the long-recognized "cytokine storm" that occurs early after donor graft infusion, cytokines and their receptors have been tested as potential aGVHD biomarkers. ${ }^{56}$ Noteworthy biomarkers that have emerged from these studies are summarized following.

In many studies, soluble IL-2 receptor $\alpha$ chain (sIL-2R $\alpha$ ) concentrations were found to be increased in aGVHD patients. ${ }^{57-61}$ In two studies, concentrations of IL-18 were closely correlated to IL-2R $\alpha .{ }^{60,62}$ IL-2R $\alpha$ concentrations were also correlated with GVHD severity. ${ }^{60}$ However, some of these studies found that sIL-2R $\alpha$ concentrations were also increased in patients with other transplantation-related complications (TRCs), such as veno-occlusive disease and sepsis. ${ }^{57}$

Similarly, TNF $\alpha$ and its receptors, particularly TNFR1, have been implicated in the pathology of aGVHD; their concentrations were found elevated in aGVHD patients compared with patients without GVHD. ${ }^{63-67}$ The same precautions used to evaluate sIL-2R $\alpha$ concerning its elevation in other TRCs should be applied to TNF $\alpha$ and TNFR1. The roles of TNF $\alpha /$ TNFR1 and IL-2/IL-2R $\alpha$ in aGVHD pathogenesis are supported by evidence that suggests that antibodies directed against TNF $\alpha$ or TNFR1, or IL-2/IL-2R $\alpha$, are effective therapies for steroid-refractory aGVHD. ${ }^{68}$

C-reactive protein (CRP), a nonspecific inflammatory protein, was found to be increased in patients with GVHD, as was IL-6, the main cytokine involved in inducing the release of CRP. ${ }^{66,69-71}$

Concentrations of IL-8 were clearly correlated with aGVHD in one study by Uguccioni et al. ${ }^{72}$ However, Schots et $a l^{66}$ showed that IL- 8 is released in all types of TRC, rather than specifically in aGVHD. Similarly, increases in IL-8 and other cytokines (eg, IL-6, IL-10, and IL-18) were not confirmed as indicators of aGVHD in a study that only included patients receiving a reduced-intensity conditioning regimen. ${ }^{73}$ However, this same study found IL-12 to be elevated in association with aGVHD development. ${ }^{73}$

Chemokines and chemokine receptors implicated in the pathology of GVHD, particularly in the migration of immune cells from lymphoid organs to target organs, have also found to be elevated in patients with aGVHD. ${ }^{74,75}$

Hepatocyte growth factor (HGF) is a multifunctional cytokine that is secreted by mesenchymal cells and acts primarily on cells of epithelial origin. Okamoto et al ${ }^{76}$ observed increased serum HGF concentrations in patients who developed aGVHD compared with patients who did not develop the disease; these authors also found that these increased HGF concentrations correlated significantly with the severity of aGVHD. HGF appears to belong to a different category of biomarkers, representing a physiologic response to GVHD damage. In this respect, HGF seems similar to cytokeratin-18 (KRT18) fragments, markers of epithelial apoptosis that have been associated with intestinal and hepatic GVHD damage. ${ }^{77}$ However, HGF possesses antiapoptotic properties and acts as mitogen for hepatocytes, enhancing liver repair and regeneration; HGF administration has been shown to prevent aGVHD in a murine model. ${ }^{78} \mathrm{HGF}$ would therefore appear not only to indicate the extent of target organ damage from GVHD, but may reflect the physiologic response intended to limit further damage from GVHD.

Four studies have identified the proteomic pattern of aGVHD using MS-based approaches on small samples without validation on independent sets of patients; one group of investigators identified a pattern that was validated in a larger study. Imanguli et $\mathrm{al}^{41}$ employed complementary proteomic techniques (eg, SELDI-TOF MS, 2D differential gel electrophoresis, and ELISA) to investigate the salivary 


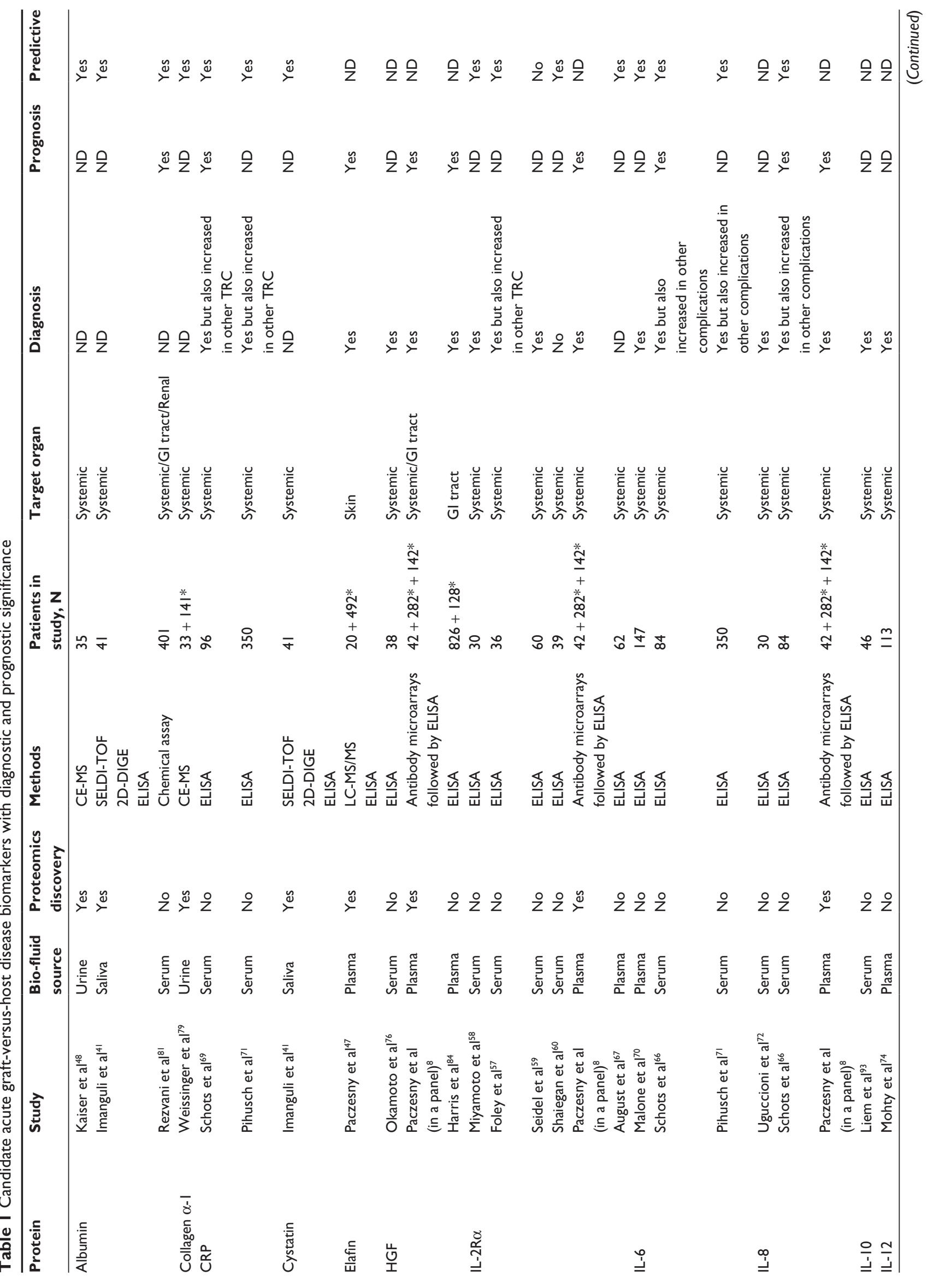




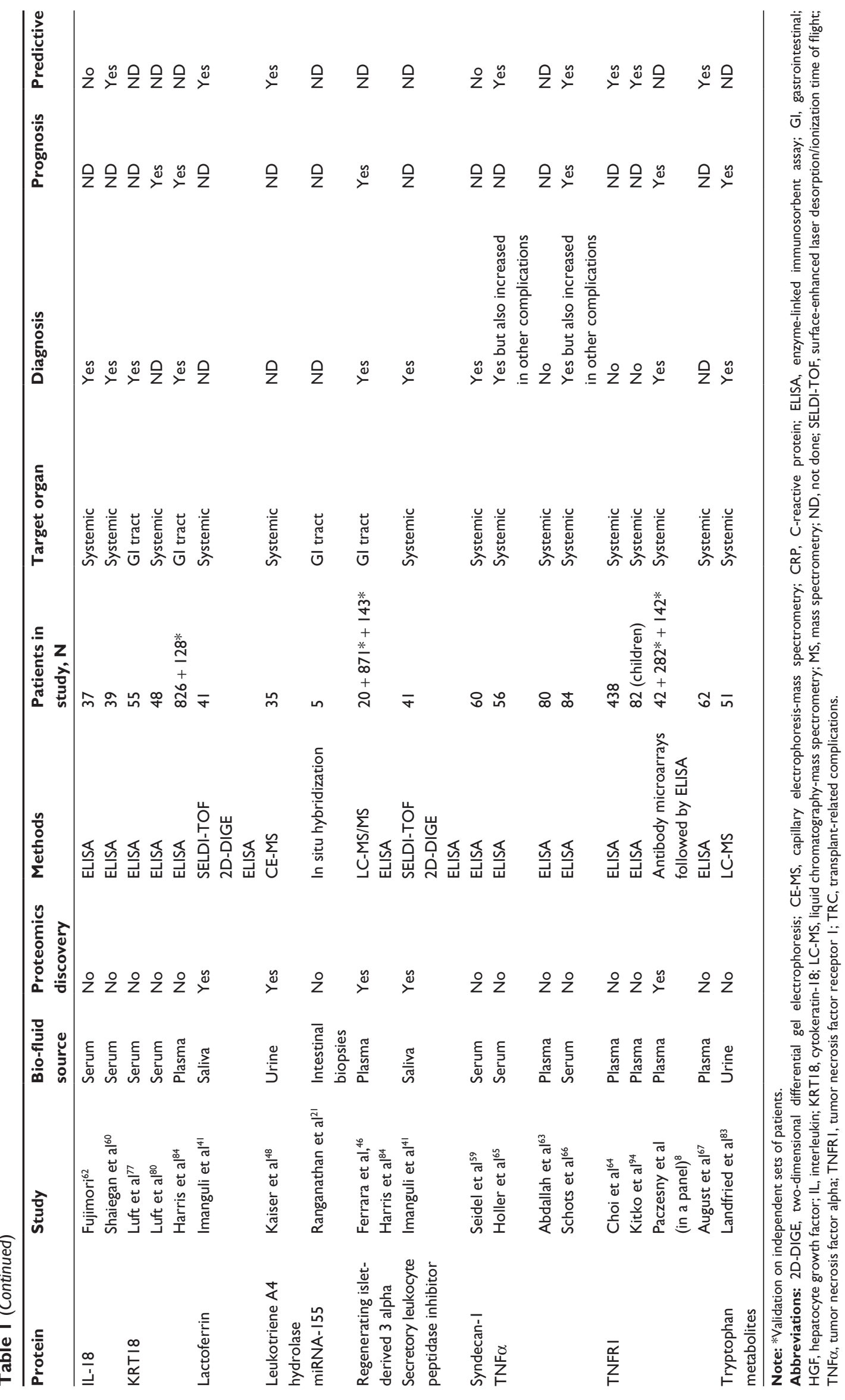


proteome of 41 patients undergoing allo-HSCT. Hori et $\mathrm{al}^{74}$ used the SELDI technique to screen for plasma proteins specific for aGVHD in a mouse model. In that study, one peak, identified as CCL8, appeared to distinguish GVHD plasma from non-GVHD plasma and was selected for further analysis. Srinivasan et $\mathrm{al}^{40}$ used SELDI-TOF MS and found an aGVHD-specific peptide pattern in training samples that was then validated in an independent set of aGVHD samples obtained on the day of onset of aGVHD symptoms. Kaiser et $\mathrm{al}^{48}$ employed capillary electrophoresis MS to identify peptide patterns in urine samples as early indicators of aGVHD development. Two prominent GVHD-indicative polypeptides were identified as a $1.85 \mathrm{kDa}$ peptide from the leukotriene A4 hydrolase and a $1.83 \mathrm{kDa}$ peptide from albumin. ${ }^{48}$ This aGVHD-specific peptide set pattern was used to screen 63 samples collected from 33 patients after allo-HSCT. ${ }^{79}$ A subsequent blind evaluation of 599 samples from 141 patients enabled the prediction of aGVHD before clinical symptoms presented, with a sensitivity of $83.1 \%$ and a specificity of $75.6 \%$. Using MS/MS, three of the 31 peptides that contributed to the aGVHD pattern were identified as fragments from collagen $\alpha-1$ chain I (downregulated) and $\alpha-1$ chain III (upregulated).

Paczesny et al identified and validated a panel of proteins using an antibody microarray. ${ }^{8}$ To validate this aGVHD biomarker panel, the samples were randomly divided into a training set of 282 patients and a validation set of 142 patients, which is currently one of the most reliable approaches for validation. This approach identified and validated a four-protein biomarker panel (IL-2R $\alpha$, TNFR1, IL-8, and HGF) with high specificity for GVHD diagnosis. Due to the large size of this study, the first demonstration that these biomarkers are associated with GVHD clinical outcomes and prognosis was provided.

Few of these studies have looked at prognostic information, such as NRM, primarily due to small sample size. An exception is the recent study from Luft et $\mathrm{al}^{80}$ that measured KRT18 along with markers of endothelial dysfunction (eg, angiopoietin-2, vascular endothelial growth factor, and thrombomodulin) and found that steroid-refractory GVHD patients were not exposed to an overwhelming $\mathrm{T}$ cell attack but rather to a progressive microangiopathy that led to organ failure.

Again due to small sample size, few of these studies looked at the predictive value of these biomarkers. Studies measuring the kinetics of cytokine concentrations during the first month post-HSCT showed that monitoring IL-2R $\alpha$ and TNFR1 concentrations during the engraftment period allows for early detection of aGVHD. The mean serum IL-2R $\alpha$ concentration appears to increase during the second week, and the mean serum TNFR1 concentration increases during the first 2 weeks following transplantation in patients with aGVHD. ${ }^{57,58,60,64-66}$ Recently, August et al ${ }^{67}$ reported that sIL-2R, sTNFR1, and sCD8 had high predictive values for aGVHD occurrence. Using these three markers, the authors demonstrated the feasibility of detecting severe aGVHD prior to the appearance of clinical symptoms.

A recent and important study by Rezvani et $\mathrm{al}^{81}$ found that a decrease of $0.5 \mathrm{~g} / \mathrm{dL}$ in serum albumin from the pretransplantation baseline level to the onset of treatment for $\mathrm{aGVHD}$ predicted the subsequent development of grade III/IV aGVHD and overall survival at 6 months after initiation of aGVHD treatment in a cohort of 401 patients with aGVHD grades II-IV after reduced-intensity HSCT. Because the measurement of serum albumin concentration is inexpensive and readily available, the authors suggest incorporating albumin into the set of other validated biomarkers to further improve prediction of aGVHD severity and mortality.

Induction of indoleamine 2,3-dioxygenase, the rate-limiting enzyme in tryptophan degradation along the kynurenine pathway, functions in a potent immunoregulatory loop and was found to be involved in the pathology of GVHD. ${ }^{82}$ Recently, one group used LC-MS/MS to measure major tryptophan metabolites, such as quinolinic acid and kynurenine, in serial urine specimens from 51 patients and found that surviving patients had significantly lower metabolite levels on days 28 , 42, and 90 following HSCT compared with patients dying of GVHD. Kynurenine levels directly correlated with severity and clinical course of GVHD. ${ }^{83}$

\section{Target-specific biomarkers of aGVHD}

Clinical symptoms of the skin (eg, maculopapular rash) and GI tract (eg, nausea, diarrhea) caused by GVHD can be difficult to distinguish from other causes (eg, infectious, drug induced). Thus, biomarkers that are GVHD- and targetspecific may improve the diagnosis of complications postHSCT. Because biomarkers present at the time of GVHD diagnosis might differ between target-specific GVHD, identification of biomarkers that are specific for GVHD target organs might improve the diagnostic and prognostic values of the systemic panel.

As mentioned, KRT18 has been the first target-specific marker of GVHD that has been associated with intestinal and hepatic GVHD damage. ${ }^{77}$

By comparing plasma pooled from ten patients with skin-specific GVHD with that from ten patients without 
GVHD in one intact protein analysis system experiment versus plasma pooled from ten patients with GI tract-specific GVHD compared with ten controls in a second intact protein analysis system experiment, thousands of spectra and hundreds of proteins were analyzed, identified, and quantified.$^{47}$ In that study, by Paczesny et al, elafin emerged as the lead biomarker candidate for skin GVHD detection at the time of clinical diagnosis. It was shown that plasma elafin concentrations in samples from 492 patients had significant diagnostic and prognostic value, including long-term survival, as a biomarker for skin GVHD. Compared with the four previously reported systemic diagnostic biomarkers of aGVHD, elafin was shown to be the single best discriminator for the diagnosis of GVHD in patients with a rash. These data also provide a proof-of-principle demonstration that biomarkers of disease related, tissue specific changes can be detected in patient plasma.

Using the same proteomics strategy, Ferrara et $\mathrm{al}^{46}$ discovered 74 proteins with increased ratios (ie, heavy to light) in patients with GI GVHD, five of which were of GI origin. Regenerating-islet-derived-3-alpha (REG3 $\alpha$ ) was the lead candidate and was validated as a biomarker of lower GI GVHD using ELISA. The authors subsequently validated REG3 $\alpha$ in two independent sets totaling 1014 patients from three different centers. This marker provides important prognostic information, including response to GVHD treatment and survival. In a follow-up study, ${ }^{84}$ REG3 $\alpha$ was compared with KRT18 and HGF, previously identified as GI GVHD markers. REG3 $\alpha$ showed that its diagnostic precision for lower GI GVHD was higher than the other two GI GVHD markers.

Based on the hypothesis that miRNA-155 is upregulated during $\mathrm{T}$ cell activation, it was recently demonstrated to be involved in the modulation of aGVHD. Upregulation of miRNA-155 was shown in intestinal biopsies from patients with pathologic evidence of intestinal aGVHD. ${ }^{21}$ Circulating levels of miRNA-155 in patients with aGVHD were not evaluated. Thus, miRNA-155 might be a potential future biomarker as well as an interesting target for therapeutic intervention in aGVHD.

There are also shortcomings in the prediction of the response to GVHD therapy. Recently, Luft et $\mathrm{al}^{80}$ showed that KRT18 and markers of endothelial dysfunction are elevated in steroid-refractory GVHD patients. REG3 $\alpha$ - as noted, a GI-specific GVHD marker - measured at onset of GI GVHD predicted response to treatment at 4 weeks. ${ }^{83}$ Levine et $\mathrm{al}^{85}$ further measured their six previously validated diagnostic biomarkers of GVHD from samples prospectively obtained at the initiation of treatment, day 14, and day 28 , in a multicenter, randomized, four-arm Phase II clinical trial for newly diagnosed aGVHD. It was found that at each of three time points - GVHD onset, 2 weeks into treatment, and 4 weeks into treatment - a panel of six biomarkers predicted the important clinical outcomes of day 28 post-therapy nonresponse and mortality at day 180 from onset..$^{85}$

\section{GVHD biomarkers and personalized medicine}

Given the progress being made in GVHD biomarker identification and validation, it is not surprising that clinical trial design will begin incorporating biomarkers. Targetspecific diagnostic biomarkers that can differentiate skin GVHD from other rashes and GI GVHD from other forms of enteritis will allow replacement of invasive biopsies. First, a simple observational trial during which samples and biopsies will be taken at onset of GVHD should be performed. During this trial, physicians will treat according to symptoms and perform biopsies as usual. A retrospective analysis of the samples with different thresholds of biomarkers will determine whether the biomarkers can replace the invasive biopsies as well as the best threshold. If this study concludes that the biomarker does as well as the biopsies, the next step would be a randomized interventional trial in which half of the patients are treated according to the biopsy results and the other half treated according to the biomarker results; the development of GVHD and other outcomes will then be evaluated.

Another potential clinical application of GVHD biomarkers is to use them to stratify patients based on risk at the time of GVHD onset before initiation of therapy. GI GVHD is considered a high-risk feature for mortality; however, given the absence of further risk stratification, the standard of care for all patients with GI GVHD is the prompt initiation of systemic steroid treatment, with the addition of secondline agents reserved for patients who fail initial therapy. Unfortunately, most patients who require second-line therapy die, highlighting the need for refinement of risk beyond what the current grading system provides. Ferrara et $\mathrm{al}^{46}$ recently developed a risk stratification algorithm for patients with new onset GI GVHD that incorporates clinical stage, histologic grade, and plasma levels of the newly discovered GI GVHD biomarker, REG3 $\alpha$. This easy to use algorithm assigns one equal-weight point to each of the three individual risk factors: clinical stage $>1$, histologic grade $>3$, and REG3 $\alpha$ concentration $>151 \mathrm{ng} / \mathrm{mL}$. Patients with two or more risk factors at onset were less likely to respond to treatment, which 
translates into highly significant differences in NRM. Patients with two or three risk factors (ie, high risk) at the onset of clinical manifestations of GI GVHD experience 1-year NRM rates of $71 \%$, whereas patients who present with no or one risk factor (standard risk) experience 1-year NRM rates of 30\% $(P<0.0001) .{ }^{46}$

Early identification of patients at high risk for steroid unresponsiveness may permit alternative testing or additional therapies before the development of refractory disease. Equally important is the identification of low-risk patients who will respond well to treatment. These patients may tolerate a more rapid tapering of steroid regimens to reduce long-term toxicity, infections, and loss of the GVT effect. The ability to identify patients at high risk for GVHD early in their transplant and treatment course has important therapeutic consequences, including more stringent monitoring and/or preemptive interventions. To determine whether Paczesny et al's ${ }^{8,46,47}$ validated biomarkers could predict GVHD before the appearance of clinical symptoms, the authors evaluated the four most informative biomarkers (ie, IL2R- $\alpha$, TNFR1, elafin, and REG3 $\alpha$ ) in samples from 513 patients who had undergone unrelated HSCT and had not yet developed GVHD. Concentrations of each biomarker were assessed at days 7 and 14 post-HSCT. Measurement of a biomarker panel pre-HSCT predicted grade II-IV GVHD with a specificity of $75 \%$ and sensitivity of $57 \% .^{86}$

The success of preemption must include not only a reduction in the incidence of GVHD, but also in infectious complications and relapse. Ultimately, a randomized trial will be needed to assess the effectiveness of GVHD preemption.

\section{Limitations to overcome for large-scale usefulness of GVHD biomarkers}

Unfortunately, there are still limitations to overcome before GVHD biomarkers can be used widely in all BMT centers. Indeed, GVHD biomarker studies have mostly been performed in T cell-repleted HSCT. Since the onset of GVHD is closely linked with the donor T cell population and major or minor HLA disparities, it is not surprising to have an overrepresentation of high biomarker concentrations in recipients from HLA mismatch and unrelated donors. ${ }^{8,46,47}$ The type of conditioning given (full [including total body irradiation-based] or reduced) also seems to have an impact on biomarker levels such as that of TNFR1. ${ }^{86}$ None of the large published studies has included T cell-depleted HSCT (in vitro $\mathrm{T}$ cell depletion of the graft or in vivo through administration of antithymocyte globulin). Differences would be expected to be observed between T cell-repleted and T celldepleted HSCT for the reason evoked above. In addition, none of these biomarkers has been studied in large cohorts of recipients receiving umbilical cord blood transplant. Due to the increased number of double-cord transplants performed and the high rate of grade II-IV GVHD observed in these cohorts, ${ }^{87-89}$ it has become even more crucial to study biomarkers in these patients. Another possibility for differences is the diverse type of prophylaxis administered, as some are known to be less responsible for inducing GVHD, such as the combination of sirolimus, tacrolimus, and lowdose methotrexate. ${ }^{90}$ All of these parameters will need to be evaluated in large-scale multicenter studies as detailed following.

Another step required is to determine which time point or which combination of different time point measurements will be most useful. This will be best achieved by realizing serial measurements during the first month post-HSCT for T cell-repleted HSCT. ${ }^{8,46,47}$ So far, only few small published studies have looked at the kinetics of cytokines during the first month post-HSCT. ${ }^{58,66,91}$ However, there are ongoing studies to address this point that have currently only been published as abstracts. ${ }^{86}$

\section{Future prospects for GVHD biomarkers}

Future directions include a blinded evaluation of these biomarkers with samples collected in a multicenter prospective study. Ideally, this requires a multicenter cohort, which would be indispensible to the reduction of center effects and to the successful design of subsequent trials. Further, such a study would be ideally performed through an institution such as the Blood and Marrow Transplant Clinical Trials Network to both establish a unique resource for BMT clinicians and a national resource for investigators to explore BMT. However, this endeavor will be expensive and might include some variation in collection and interpretation of clinical data. Such multicenter prospective trial validation is important because the algorithm should take into account the variability between centers (center effect) and the individual risks related to known risk factors, such as age, HLA match, donor source (particularly cord blood), and conditioning regimen, including $\mathrm{T}$ cell depletion (in vivo or in vitro). The statistical model should use the methods discussed previously. Ideally, a single formula should be able to be developed to predict a patient's risk for aGVHD, allowing 
for cutting-edge personalized medicine. The ideal formula will be as simple as possible. In the best-case scenario, a single marker at a single time point and few transplantation risk factors (eg, conditioning intensity, cord blood source, and use of $\mathrm{T}$ cell depletion) would be investigated. Next, a trial of preemptive therapy for aGVHD using the formula would be initiated.

Over the past 30 years, therapeutic approaches for aGVHD have largely been limited to the nonspecific targeting of effector cells. As a result, steroids remain the first-line treatment for patients presenting with aGVHD symptoms. Biomarkers represent promising targets for new therapeutics. In addition, it is proposed that the discovery of aGVHD-specific drugs based on biomarkers will target the appropriate effector $\mathrm{T}$ cells to both increase efficacy and lower toxicity. This approach represents the first step in a continuum of research that is expected to lead to the development of pharmacologic strategies to specifically treat GVHD. One direct outcome of this proposal will be the establishment of clinical trials using both biomarkers for risk stratification and new drugs for treatment in high-risk populations.

So far, development of biomarkers post-HSCT has focused on aGVHD biomarkers. However, future efforts in biomarker discovery and validation would be particularly valuable for cGVHD biomarkers, whose current consensus criteria are labor intensive and still not validated prospectively.

\section{Conclusion}

Proteomics is a revolutionary field that includes detection technologies for proteins, molecules that are the most proximal to the real-time pathophysiology of alloreactivity. In a short time, the use of proteomics has led to the identification of novel mechanisms of allo-HSCT, which were unlikely to have been discovered by traditional hypothesis-driven research. A promising proteomic approach is to use protein biomarkers in risk stratification to better employ current disease treatment modalities.

Furthermore, the biomarker findings presented in this review have the potential for exploring targeted therapeutics. Unlike genes, protein levels may be influenced by several posttranscriptional modifications and other factors, such as the cytokine milieu. The principal barrier that must be circumvented is the validation of biomarker concentrations in different types of allo-HSCT settings (eg, conditioning intensity, donor sources [particularly cord blood and T celldepleted grafts]). Achieving this aim will require a much larger validation study, ideally in a multicenter prospective trial. Once an algorithm for each setting is established, personalized medicine will be possible.

\section{Acknowledgment}

The author is supported by grants from the NIH, RC1HL101102 and P01-CA039542, and is an investigator of the Eric Hartwell Research Fund and the Amy Strelzer Manasevit Research Program.

\section{Disclosure}

The author declares no conflicts of interest in this work.

\section{References}

1. Martin PJ, Schoch G, Fisher L, et al. A retrospective analysis of therapy for acute graft-versus-host disease: initial treatment. Blood. 1990;76(8):1464-1472.

2. Sullivan KM, Mori M, Sanders J, et al. Late complications of allogeneic and autologous marrow transplantation. Blood Marrow Transplant. 1992;10 Suppl 1:127-134.

3. Filipovich AH, Weisdorf D, Pavletic S, et al. National Institutes of Health consensus development project on criteria for clinical trials in chronic graft-versus-host disease: I. Diagnosis and staging working group report. Biol Blood Marrow Transplant. 2005;11(12):945-956.

4. Griffith LM, Pavletic SZ, Lee SJ, Martin PJ, Schultz KR, Vogelsang GB. Chronic Graft-versus-Host Disease - implementation of the National Institutes of Health Consensus Criteria for Clinical Trials. Biol Blood Marrow Transplant. 2008;14(4):379-384.

5. Paczesny S, Hanauer D, Sun Y, Reddy P. New perspectives on the biology of acute GVHD. Blood Marrow Transplant. 2010;45(1):1-11.

6. Biomarkers Definitions Working Group. Biomarkers and surrogate endpoints: preferred definitions and conceptual framework. Clin Pharmacol Ther. 2001;69(3):89-95.

7. MowatA, Socié G. Intestinal graft-vs-host disease. In: FerraraJL, CookeKR, Deeg HJ, editors. Graft-vs-Host Disease. 3rd ed. New York, NY: Marcel Dekker; 2004:279-327.

8. Paczesny S, Krijanovski OI, Braun TM, et al. A biomarker panel for acute graft-versus-host disease. Blood. 2009;113(2):273-278.

9. Schlatzer DM, Dazard JE, Ewing RM, et al. Human biomarker discovery and predictive models for disease progression for idiopathic pneumonia syndrome following allogeneic stem cell transplantation. Mol Cell Proteomics. 2012;11(6):M111.015479.

10. Flomenberg N, Baxter-Lowe LA, Confer D, et al. Impact of HLA class I and class II high-resolution matching on outcomes of unrelated donor bone marrow transplantation: HLA-C mismatching is associated with a strong adverse effect on transplantation outcome. Blood. 2004;104(7): 1923-1930.

11. den Haan JM, Sherman NE, Blokland E, et al. Identification of a graft versus host disease-associated human minor histocompatibility antigen. Science. 1995;268(5216):1476-1480.

12. Goulmy E, Schipper R, Pool J, et al. Mismatches of minor histocompatibility antigens between HLA-identical donors and recipients and the development of graft-versus-host disease after bone marrow transplantation. N Engl J Med. 1996;334(5):281-285.

13. Murata M, Warren EH, Riddell SR. A human minor histocompatibility antigen resulting from differential expression due to a gene deletion. J Exp Med. 2003;197(10):1279-1289.

14. Kaplan DH, Anderson BE, McNiff JM, Jain D, Shlomchik MJ, Shlomchik WD. Target antigens determine graft-versus-host disease phenotype. J Immunol. 2004;173(9):5467-5475.

15. Bleakley M, Riddell SR. Molecules and mechanisms of the graft-versusleukaemia effect. Nat Rev Cancer. 2004;4(5):371-380. 
16. Bleakley M, Riddell SR. Exploiting T cells specific for human minor histocompatibility antigens for therapy of leukemia. Immunol Cell Biol. 2011;89(3):396-407.

17. Hansen JA, Chien JW, Warren EH, Zhao LP, Martin PJ. Defining genetic risk for graft-versus-host disease and mortality following allogeneic hematopoietic stem cell transplantation. Curr Opin Hematol. 2010;17(6):483-492.

18. Moss EG, Poethig RS. MicroRNAs: something new under the sun. Curr Biol. 2002;12(20):R688-R690.

19. Chen PY, Meister G. microRNA-guided posttranscriptional gene regulation. Biol Chem. 2005;386(12):1205-1218.

20. Ajit SK. Circulating microRNAs as biomarkers, therapeutic targets, and signaling molecules. Sensors (Basel). 2012;12(3):3359-3369.

21. Ranganathan $\mathrm{P}$, Heaphy CE, Costinean S, et al. Regulation of acute graft-versus-host disease by microRNA-155. Blood. 2012;119(20): 4786-4797.

22. Magenau JM, Qin X, Tawara I, et al. Frequency of CD4(+)CD25(hi) FOXP3(+) regulatory $T$ cells has diagnostic and prognostic value as a biomarker for acute graft-versus-host-disease. Biol Blood Marrow Transplant. 2010;16(7):907-914.

23. Teshima T, Maeda Y, Ozaki K. Regulatory T cells and IL-17-producing cells in graft-versus-host disease. Immunotherapy. 2011;3(7): $833-852$.

24. Zinöcker S, Sviland L, Dressel R, Rolstad B. Kinetics of lymphocyte reconstitution after allogeneic bone marrow transplantation: markers of graft-versus-host disease. J Leukoc Biol. 2011;90(1):177-187.

25. Koreth J, Matsuoka K, Kim HT, et al. Interleukin-2 and regulatory T cells in graft-versus-host disease. $N$ Engl J Med. 2011;365(22):2055-2066.

26. Lau J, Sartor M, Bradstock KF, Vuckovic S, Munster DJ, Hart DN. Activated circulating dendritic cells after hematopoietic stem cell transplantation predict acute graft-versus-host disease. Transplantation. 2007;83(7):839-846

27. Akhtari M, Giver CR, Ali Z, et al. Receiver operating characteristic curve analysis of circulating blood dendritic cell precursors and $\mathrm{T}$ cells predicts response to extracorporeal photopheresis in patients with chronic graft-versus-host disease. Transfusion. 2010;50(11):2424-2431.

28. Levine JE, Paczesny S, Sarantopoulos S. Clinical applications for biomarkers of acute and chronic graft-versus-host disease. Biol Blood Marrow Transplant. 2012;18(1 Suppl):S116-S124.

29. Court M, Selevsek N, Matondo M, et al. Toward a standardized urine proteome analysis methodology. Proteomics. 2011;11(6):1160-1171.

30. Rai AJ, Gelfand CA, Haywood BC, et al. HUPO Plasma Proteome Project specimen collection and handling: towards the standardization of parameters for plasma proteome samples. Proteomics. 2005;5(13): 3262-3277.

31. Anderson NL, Anderson NG. The human plasma proteome: history, character, and diagnostic prospects. Mol Cell Proteomics. 2002;1(11): 845-867.

32. Tirumalai RS, Chan KC, Prieto DA, Issaq HJ, Conrads TP, Veenstra TD Characterization of the low molecular weight human serum proteome. Mol Cell Proteomics. 2003;2(10):1096-1103.

33. Thongboonkerd V. Recent progress in urinary proteomics. Proteomics Clin Appl. 2007;1(8):780-791.

34. Schaub S, Rush D, Wilkins J, et al. Proteomic-based detection of urine proteins associated with acute renal allograft rejection. J Am Soc Nephrol. 2004;15(1):219-227.

35. Mann M, Jensen ON. Proteomic analysis of post-translational modifications. Nat Biotechnol. 2003;21(3):255-261.

36. Wild D, editor. The Immunoassay Handbook. 3rd ed. Oxford: Elsevier; 2005.

37. Schweitzer B, Roberts S, Grimwade B, et al. Multiplexed protein profiling on microarrays by rolling-circle amplification. Nat Biotechnol. 2002;20(4):359-365.

38. Boja E, Hiltke T, Rivers R, et al. Evolution of clinical proteomics and its role in medicine. J Proteome Res. 2011;10(1):66-84.

39. Rodland KD. Proteomics and cancer diagnosis: the potential of mass spectrometry. Clin Biochem. 2004;37(7):579-583.
40. Srinivasan R, Daniels J, Fusaro V, et al. Accurate diagnosis of acute graft-versus-host disease using serum proteomic pattern analysis. Exp Hematol. 2006;34(6):796-801.

41. Imanguli MM, Atkinson JC, Harvey KE, et al. Changes in salivary proteome following allogeneic hematopoietic stem cell transplantation. Exp Hematol. 2007;35(2):184-192.

42. O'Farrell PH. High resolution two-dimensional electrophoresis of proteins. J Biol Chem. 1975;250:4007-4021.

43. Marouga R, David S, Hawkins E. The development of the DIGE system: 2D fluorescence difference gel analysis technology. Anal Bioanal Chem. 2005;382(3):669-678.

44. Wang H, Clouthier SG, Galchev V, et al. Intact-protein-based high-resolution three-dimensional quantitative analysis system for proteome profiling of biological fluids. Mol Cell Proteomics. 2005;4(5):618-625.

45. Chen CH, Budas GR, Churchill EN, Disatnik MH, Hurley TD, Mochly-Rosen D. Activation of aldehyde dehydrogenase-2 reduces ischemic damage to the heart. Science. 2008;321(5895):1493-1495.

46. Ferrara JL, Harris AC, Greenson JK, et al. Regenerating islet-derived 3 alpha is a biomarker of gastrointestinal graft-versus-host disease. Blood. 2011;118(25):6702-6708.

47. Paczesny S, Braun TM, Levine JE, et al. Elafin is a biomarker of graftversus-host disease of the skin. Sci Transl Med. 2010;2(13):13ra12.

48. Kaiser T, Kamal H, Rank A, et al. Proteomics applied to the clinical follow-up of patients after allogeneic hematopoietic stem cell transplantation. Blood. 2004;104(2):340-349.

49. Brewis IA, Brennan P. Proteomics technologies for the global identification and quantification of proteins. Adv Protein Chem Struct Biol. 2010;80:1-44.

50. Faca V, Coram M, Phanstiel D, et al. Quantitative analysis of acrylamide labeled serum proteins by LC-MS/MS. J Proteome Res. 2006;5(8):2009-2018.

51. Hanash SM, Pitteri SJ, Faca VM. Mining the plasma proteome for cancer biomarkers. Nature. 2008;452(7187):571-579.

52. Elliott MH, Smith DS, Parker CE, Borchers C. Current trends in quantitative proteomics. J Mass Spectrom. 2009;44(12):1637-1660.

53. Michalski A, Damoc E, Lange O, et al. Ultra high resolution linear ion trap Orbitrap mass spectrometer (Orbitrap Elite) facilitates top down LC MS/MS and versatile peptide fragmentation modes. Mol Cell Proteomics. 2012;11(3):O111.013698.

54. MacLean B, Eng JK, Beavis RC, McIntosh M. General framework for developing and evaluating database scoring algorithms using the TANDEM search engine. Bioinformatics. 2006;22(22):2830-2832.

55. Kitteringham NR, Jenkins RE, Lane CS, Elliott VL, Park BK. Multiple reaction monitoring for quantitative biomarker analysis in proteomics and metabolomics. J Chromatogr B Analyt Technol Biomed Life Sci. 2009;877(13):1229-1239.

56. Toubai T, Tanaka J, Paczesny S, Shono Y, Reddy P, Imamura M. Role of cytokines in the pathophysiology of acute graft-versus-host disease (GVHD): are serum/plasma cytokines potential biomarkers for diagnosis of acute GVHD following allogeneic hematopoietic cell transplantation (Allo-HCT)? Curr Stem Cell Res Ther. 2012;7(3):229-239.

57. Foley R, Couban S, Walker I, et al. Monitoring soluble interleukin-2 receptor levels in related and unrelated donor allogenic bone marrow transplantation. Blood Marrow Transplant. 1998;21(8):769-773.

58. Miyamoto T, Akashi K, Hayashi S, et al. Serum concentration of the soluble interleukin-2 receptor for monitoring acute graft-versus-host disease. Blood Marrow Transplant. 1996;17(2):185-190.

59. Seidel C, Ringdén O, Remberger M. Increased levels of syndecan-1 in serum during acute graft-versus-host disease. Transplantation. 2003; 76(2):423-426.

60. Shaiegan M, Iravani M, Babaee GR, Ghavamzadeh A. Effect of IL-18 and sIL2R on aGVHD occurrence after hematopoietic stem cell transplantation in some Iranian patients. Transpl Immunol. 2006;15(3): 223-227.

61. Visentainer JE, Lieber SR, Persoli LB, et al. Serum cytokine levels and acute graft-versus-host disease after HLA-identical hematopoietic stem cell transplantation. Exp Hematol. 2003;31(11):1044-1050. 
62. Fujimori Y. Elevated interleukin (IL)-18 levels during acute graftversus-host disease after allogeneic bone marrow transplantation. $\mathrm{Br} \mathrm{J}$ Haematol. 2000;109:652-657.

63. Abdallah AN, Boiron JM, Attia Y, Cassaigne A, Reiffers J, Iron A. Plasma cytokines in graft vs host disease and complications following bone marrow transplantation. Hematol Cell Ther. 1997;39(1): $27-32$.

64. Choi SW, Kitko CL, Braun T, et al. Change in plasma tumor necrosis factor receptor 1 levels in the first week after myeloablative allogeneic transplantation correlates with severity and incidence of GVHD and survival. Blood. 2008;112(4):1539-1542.

65. Holler E, Kolb HJ, Möller A, et al. Increased serum levels of tumor necrosis factor alpha precede major complications of bone marrow transplantation. Blood. 1990;75(4):1011-1016.

66. Schots R, Kaufman L, Van Riet I, et al. Proinflammatory cytokines and their role in the development of major transplant-related complications in the early phase after allogeneic bone marrow transplantation. Leukemia. 2003;17(6):1150-1156.

67. August KJ, Chiang KY, Bostick RM, et al. Biomarkers of immune activation to screen for severe, acute GVHD. Bone Marrow Transplant. 2011;46(4):601-604.

68. Paczesny S, Choi SW, Ferrara JL. Acute graft-versus-host disease: new treatment strategies. Curr Opin Hematol. 2009;16(6):427-436.

69. Schots R, Van Riet I, Othman TB, et al. An early increase in serum levels of C-reactive protein is an independent risk factor for the occurrence of major complications and 100-day transplant-related mortality after allogeneic bone marrow transplantation. Bone Marrow Transplant. 2002;30(7):441-446.

70. Malone FR, Leisenring WM, Storer BE, et al. Prolonged anorexia and elevated plasma cytokine levels following myeloablative allogeneic hematopoietic cell transplant. Bone Marrow Transplant. 2007;40(8): $765-772$.

71. Pihusch M, Pihusch R, Fraunberger P, et al. Evaluation of C-reactive protein, interleukin-6, and procalcitonin levels in allogeneic hematopoietic stem cell recipients. Eur J Haematol. 2006;76(2):93-101.

72. Uguccioni M, Meliconi R, Nesci S, et al. Elevated interleukin-8 serum concentrations in beta-thalassemia and graft-versus-host disease. Blood. 1993;81(9):2252-2256.

73. Mohty M, Blaise D, Faucher C, et al. Inflammatory cytokines and acute graft-versus-host disease after reduced-intensity conditioning allogeneic stem cell transplantation. Blood. 2005;106(13):4407-4411.

74. Hori T, Naishiro Y, Sohma H, et al. CCL8 is a potential molecular candidate for the diagnosis of graft-versus-host disease. Blood. 2008; 111(8):4403-4412.

75. Piper KP, Horlock C, Curnow SJ, et al. CXCL10-CXCR3 interactions play an important role in the pathogenesis of acute graft-versus-host disease in the skin following allogeneic stem-cell transplantation. Blood. 2007;110(12):3827-3832.

76. Okamoto T, Takatsuka H, Fujimori Y, Wada H, Iwasaki T, Kakishita E. Increased hepatocyte growth factor in serum in acute graft-versus-host disease. Blood Marrow Transplant. 2001;28(2):197-200.

77. Luft T, Conzelmann M, Benner A, et al. Serum cytokeratin-18 fragments as quantitative markers of epithelial apoptosis in liver and intestinal graft-versus-host disease. Blood. 2007;110(13):4535-4542.

78. Kuroiwa T, Kakishita E, Hamano T, et al. Hepatocyte growth factor ameliorates acute graft-versus-host disease and promotes hematopoietic function. J Clin Invest. 2001;107(11):1365-1373.

Current Biomarker Findings

\section{Publish your work in this journal}

Current Biomarker Findings is an international, peer-reviewed, open access journal publishing original research, reports, reviews and commentaries on all areas of biomarker research. The manuscript management system is completely online and includes a very quick and fair
79. Weissinger EM, Schiffer E, Hertenstein B, et al. Proteomic patterns predict acute graft-versus-host disease after allogeneic hematopoietic stem cell transplantation. Blood. 2007;109(12):5511-5519.

80. Luft T, Dietrich S, Falk C, et al. Steroid-refractory GVHD: T-cell attack within a vulnerable endothelial system. Blood. 2011;118(6): 1685-1692.

81. Rezvani AR, Storer BE, Storb RF, et al. Decreased serum albumin as a biomarker for severe acute graft-versus-host disease after reducedintensity allogeneic hematopoietic cell transplantation. Biol Blood Marrow Transplant. 2011;17(11):1594-1601.

82. Reddy P, Sun Y, Toubai T, et al. Histone deacetylase inhibition modulates indoleamine 2,3-dioxygenase-dependent DC functions and regulates experimental graft-versus-host disease in mice. $J$ Clin Invest. 2008;118(7):2562-2573.

83. Landfried K, Zhu W, Waldhier MC, et al. Tryptophan catabolism is associated with acute GVHD after human allogeneic stem cell transplantation and indicates activation of indoleamine 2,3-dioxygenase. Blood. 2011;118(26):6971-6974.

84. Harris AC, Ferrara JL, Braun TM, et al. Plasma biomarkers of lower gastrointestinal and liver acute GVHD. Blood. 2012;119(12): 2960-2963.

85. Levine JE, Logan BR, Wu J, et al. Acute graft-versus-host disease biomarkers measured during therapy can predict treatment outcomes: a Blood and Marrow Transplant Clinical Trials Network study. Blood. 2012;119(16):3854-3860.

86. Paczesny S, Braun T, Lugt MV, et al. Three biomarker panel at day 7 and 14 can predict development of Grade II-IV acute graft-versus-host disease. In: ASH Annual Meeting Abstracts. 52nd ASH Annual Meeting and Exposition: 2010 Dec 4-7; Orlando, FL. Blood. 2010;116:675.

87. Andersen J, Heilmann C, Jacobsen N, Nielsen C, Bendtzen K, Müller K. Differential effect of conditioning regimens on cytokine responses during allogeneic stem cell transplantation. Bone Marrow Transplant. 2006;37(7):635-640

88. Sauter C, Barker JN. Unrelated donor umbilical cord blood transplantation for the treatment of hematologic malignancies. Curr Opin Hematol. 2008;15(6):568-575.

89. Brunstein CG, Fuchs EJ, Carter SL, et al. Alternative donor transplantation after reduced intensity conditioning: results of parallel phase 2 trials using partially HLA-mismatched related bone marrow or unrelated double umbilical cord blood grafts. Blood. 2011;118(2):282-288.

90. Brunstein CG, Gutman JA, Weisdorf DJ, et al. Allogeneic hematopoietic cell transplantation for hematologic malignancy: relative risks and benefits of double umbilical cord blood. Blood. 2010;116(22):4693-4699.

91. Alyea EP, Li S, Kim HT, et al. Sirolimus, tacrolimus, and low-dose methotrexate as graft-versus-host disease prophylaxis in related and unrelated donor reduced-intensity conditioning allogeneic peripheral blood stem cell transplantation. Biol Blood Marrow Transplant. 2008;14(8):920-926.

92. Grimm J, Zeller W, Zander AR. Soluble interleukin-2 receptor serum levels after allogeneic bone marrow transplantations as a marker for GVHD. Blood Marrow Transplant. 1998;21(1):29-32.

93. Liem L, van Houwelingen H, Goulmy E. Serum cytokine levels after HLA-identical bone marrow transplantation. Transplantation. 1998;66:863-871.

94. Kitko CL, Paczesny S, Yanik G, et al. Plasma elevations of tumor necrosis factor-receptor- 1 at day 7 postallogeneic transplant correlate with graft-versus-host disease severity and overall survival in pediatric patients. Biol Blood Marrow Transpl. 2008;14:759-765.

\section{Dovepress}

peer-review system. Visit http://www.dovepress.com/testimonials.php to read real quotes from published authors. 